\title{
Infrared Pump-Probe Spectroscopy of Plasmons in Graphene and Semiconductors
}

\author{
M. Wagner ${ }^{1}$, Z. Fei $^{1}$, A. S. McLeod ${ }^{1}$, S. J. Maddox ${ }^{2}$, A. S. Rodin ${ }^{1}$, W. Bao ${ }^{3}$, E. G. Iwinski ${ }^{1}$, Z. Zhao ${ }^{4}$, M. $^{2}$ \\ Goldflam $^{1}$, M. Liu ${ }^{1}$, G. Dominguez ${ }^{5,6}$, M. Thiemens ${ }^{6}$, M. M. Fogler ${ }^{1}$, A. H. Castro-Neto ${ }^{7}$, C. N. Lau ${ }^{4}$, S.

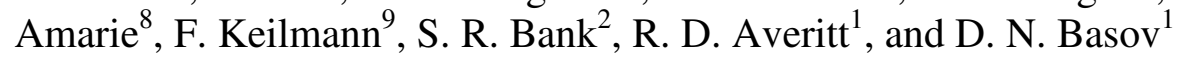 \\ ${ }^{1 .}$ University of California San Diego, Department of Physics, La Jolla, California 92093. \\ ${ }^{2}$ The University of Texas at Austin, Microelectronics Research Center, Austin, TX 78758. \\ ${ }^{3 .}$ University of Maryland, Materials Research Science and Engineering Center, College Park, Maryland \\ 20742. \\ 4. University of California, Department of Physics and Astronomy, Riverside, California 92521. \\ ${ }^{5 .}$ California State University, Department of Physics, San Marcos, San Marcos, California 92096. \\ ${ }^{6 .}$ University of California San Diego, Department of Chemistry and Biochemistry, La Jolla, California \\ 92093. \\ ${ }^{7}$ Graphene Research Centre and Department of Physics, National University of Singapore, 117542, \\ Singapore. \\ ${ }^{8 .}$ Neaspec GmbH, Bunsenstr. 5, 82152 Martinsried, München, Germany. \\ ${ }^{9}$ Ludwig-Maximilians-Universität and Center for Nanoscience, 80539 München, Germany.
}

Unraveling exciting new physics in complex novel materials requires access to both material excitations and their dynamics, thus continuously pushing ultrafast pump-probe spectroscopy to its limits. However, most of the materials whose dynamics are at the center of current attention are also known to be inhomogeneous at the nanoscale. Hence, diffraction-limited optical techniques with their inherent areaaveraging character inhibit access to characteristic time scales of nanoscopic, heterogeneous systems. Circumventing the diffraction limit, scattering scanning near-field optical microscopy (s-SNOM) is a well-established technique that enables broad-band infrared spectroscopy with the nanoscale spatial resolution. In s-SNOM backscattered light from an atomic force microscope (AFM) tip reveals the local dielectric function of a sample [1]. Previous infrared s-SNOM studies were static, utilizing primarily continuous wave $(\mathrm{CW})$ laser sources. Here, we extend s-SNOM by merging nano-spectroscopy with ultrafast pump-probe techniques and exemplify new capabilities with the time-resolved control of the plasmonic response of graphene and the semiconductor InAs.

The goal of plasmonics is to utilize electromagnetic energy on a sub-wavelength scale in form of collective surface charge oscillations. Amongst various candidates for plasmonic media graphene stands out due to its most favorable properties: ultimate energy confinement in a monoatomic layer along with easy control over its charge density via electrostatic fields. Recently, the strong coupling between Dirac plasmons and the AFM tip of a near-field microscope has proven ideal to investigate their characteristics, so far utilized for their infrared spectroscopy [2] and real space visualization [3,4]. Another interesting material class for plasmonics are semiconductors due to mature processing technologies and carrier density control via doping.

In our experiment we combine mid-infrared s-SNOM and ultrafast $100 \mathrm{fs}$, near-infrared laser excitation (Fig. 1a)) to study the time-dependent behavior of graphene [5] and InAs plasmons [6] at the nanoscale in infrared spectroscopy. For graphene we find strong pump-induced spectral changes in the infrared plasmonic response (Fig. 1b), top) around the $\mathrm{SiO}_{2}$ substrate phonon resonances at 800 and $1125 \mathrm{~cm}^{-1}$ (Fig. 1b), bottom panel). Modeling reveals that pump-induced heating of carriers up to a temperature of $2100 \mathrm{~K}$ is the dominant effect. It results in an increase in Drude weight that s-SNOM is sensitive to. In 
terms of its efficiency, optical control of graphene plasmons challenges conventional electrostatic gating (dots in Fig. 1b)), but occurs on a much faster, sub-picosecond time scale as deduced from the pumpprobe time-delay curves in Fig. 1c). Remarkably, modifying graphene's plasmonic behavior requires two orders of magnitude less pulse energy than for metal-based plasmonic structures, which can be easily achieved with a standard fiber laser at telecom wavelengths of $1.5 \mu \mathrm{m}$, as in our case. In addition, we discuss data obtained on the prototypical semiconductor InAs where the photoinduced change in the infrared originating from plasmons is an order of magnitude larger at very low control pulse fluence compared to any previous plasmonic modulator scheme. Figure 1d) shows the infrared response for different pump-probe time delays together with a Drude model that nicely fits our data. After photoexcitation a broad, low-frequency peak appears that shifts to lower frequencies as the carriers relax on a time scale of several picoseconds.

For both materials, graphene and InAs, the pump-probe spectroscopy results reveal ultrafast optical modulation with high efficiency opening thus the gate to ultrafast plasmonic devices.

\section{References:}

[1] J M Atkin et al., Adv. Phys. 61 (2012), p. 745.

[2] Z Fei et al., Nano Lett. 11 (2011), p. 4701.

[3] Z Fei et al., Nature 487 (2012), p. 82.

[4] J Chen et al., Nature 487 (2012), p. 77.

[5] M Wagner et al., Nano Lett. 14 (2014), p. 894.

[6] M Wagner et al., Nano Lett. 14 (2014), p. 4529.

a)

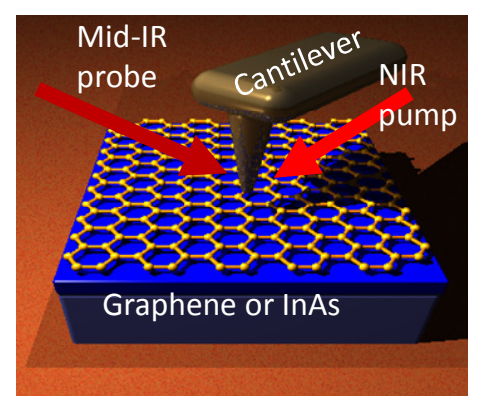

b)

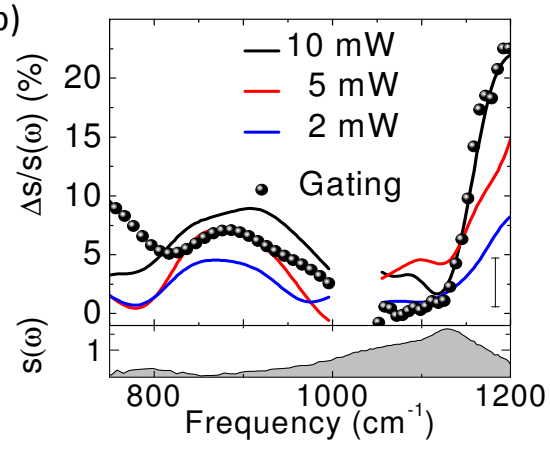

c)

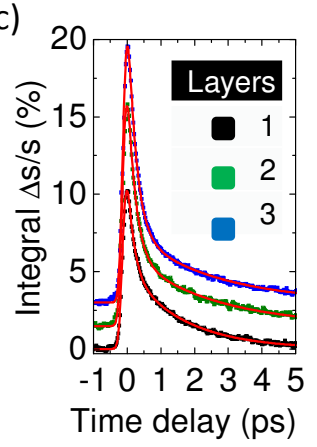

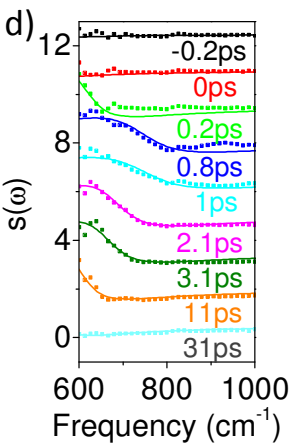

Fig 1. a) Sketch of the ultrafast NIR pump mid-IR probe experiment on exfoliated graphene or InAs. b) Relative pump-induced spectral changes for different NIR pump powers in graphene compared to electrostatic gating. The panel at the bottom shows the absolute signal without pump revealing the hybrid graphene plasmon $\mathrm{SiO}_{2}$ substrate phonon modes at 800 and $1125 \mathrm{~cm}^{-1}$. c) Ultrafast decay of the spectral changes shown in b) for single-, bi- and trilayer graphene together with biexponential decay fits (red curves). d) Photoexcited plasmons in InAs for different pump-probe delays showing ultrafast decay. Experimental data (dots) are plotted together with theory curves from a Drude model (solid lines). 